\title{
Partially coherent surface plasmon modes
}

\section{G. Martínez Niconoff \\ gmartin@inaoep.mx}

\section{P. Martínez Vara}

\section{J. Munoz-Lopez}

\section{J.C. Juárez-Morales}

\section{A. Carbajal-Dominguez}

\author{
Instituto Nacional de Astrofisica, Optica y Electronica, INAOE, Departamento de Optica, Grupo de \\ Optica Estadistica, Apdo. 51 y 216, C. P. 72000, Puebla, Mexico \\ Instituto Nacional de Astrofisica, Optica y Electronica, INAOE, Departamento de Optica, Grupo de \\ Optica Estadistica, Apdo. 51 y 216, C. P. 72000, Puebla, Mexico \\ Instituto Nacional de Astrofisica, Optica y Electronica, INAOE, Departamento de Optica, Grupo de \\ Optica Estadistica, Apdo. 51 y 216, C. P. 72000, Puebla, Mexico \\ Instituto Nacional de Astrofisica, Optica y Electronica, INAOE, Departamento de Optica, Grupo de \\ Optica Estadistica, Apdo. 51 y 216, C. P. 72000, Puebla, Mexico \\ Universidad Juárez Autonoma de Tabasco, UJAT, C.P. 86040, Tabasco, Mexico
}

Elementary long-range plasmon modes are described assuming an exponential dependence of the refractive index in the neighbourhood of the interface dielectric-metal thin film. The study is performed using coupling mode theory. The interference between two longrange plasmon modes generated that way allows the synthesis of surface sinusoidal plasmon modes, which can be considered as completely coherent generalized plasmon modes. These sinusoidal plasmon modes are used for the synthesis of new partially coherent surface plasmon modes, which are obtained by means of an incoherent superposition of sinusoidal plasmon modes where the period of each one is considered as a random variable. The kinds of surface modes generated have an easily tuneable profile controlled by means of the probability density function associated to the period. We show that partially coherent plasmon modes have the remarkable property to control the length of propagation which is a notable feature respect to the completely coherent surface plasmon mode. The numerical simulation for sinusoidal, Bessel, Gaussian and Dark Hollow plasmon modes are presented. [D01: 10.2971/jeos.2011.11009]

Keywords: surface plasmons, coherence

\section{INTRODUCTION}

Elementary surface plasmon modes [1], are nonhomogeneous electromagnetic waves propagating on the interface of a dielectric-metal media. An interesting feature of surface plasmon modes is its interaction with other structures such as nanoparticles, this interaction has been reported by several authors. Interesting physical features have been presented, for example, the spectroscopy properties of a single nanoparticle when it is deposited on different substrates [2]-[4]. Essentially, the interaction between surfacenanoparticle implies that some physical concepts must be readapted to this configuration, and in a particular manner the concept of refractive index. This is due to the fact that in the optical classical model the configuration for the change between two media makes use of an abrupt change in the refractive index avoiding the index transition. However, being a surface effect it is necessary to implement a model to describe the surface changes in the refractive index to reflect the physical reality.

For a semi-infinite media, the electric field for an elementary surface plasmon mode is

$$
E(x, z)=\left(\hat{i} a_{1}+\hat{k} a_{2}\right) \exp \{-\alpha x\} \exp \{i \beta z\}
$$

where $\beta$ is the dispersion relation function given by

$$
\beta=\frac{\omega}{c}\left(\frac{\varepsilon_{1} \varepsilon_{2}}{\varepsilon_{1}+\varepsilon_{2}}\right)^{1 / 2}
$$

$\omega$ is the frequency of light, $\alpha$ is a parameter whose value determines the decreasing rate in $x$-coordinate, $\varepsilon_{1}$ and $\varepsilon_{2}$ are the permittivity of the dielectric and conductive media respectively. In a media free of charge, the electric field must satisfy $\nabla \cdot E=0$, this condition means that parameters $\alpha_{1}, \alpha_{2}$, $\alpha$ and $\beta$ in Eq. 1 are related by

$$
a_{2}=-\frac{i \alpha a_{1}}{\beta}
$$

where $\alpha_{1}$ is a constant which depends of the excitation strength of the surface plasmon mode, more details concerning the physical implications of these parameters can be found in Raether [1]. The permittivity of the conducting media have a mathematical representation by means of a complex number; consequently the dispersion relation function $\beta$ have a complex representation too. As a consequence of the imaginary part of $\beta$, among other factors such as attenuation due to electric resistance, the elementary 


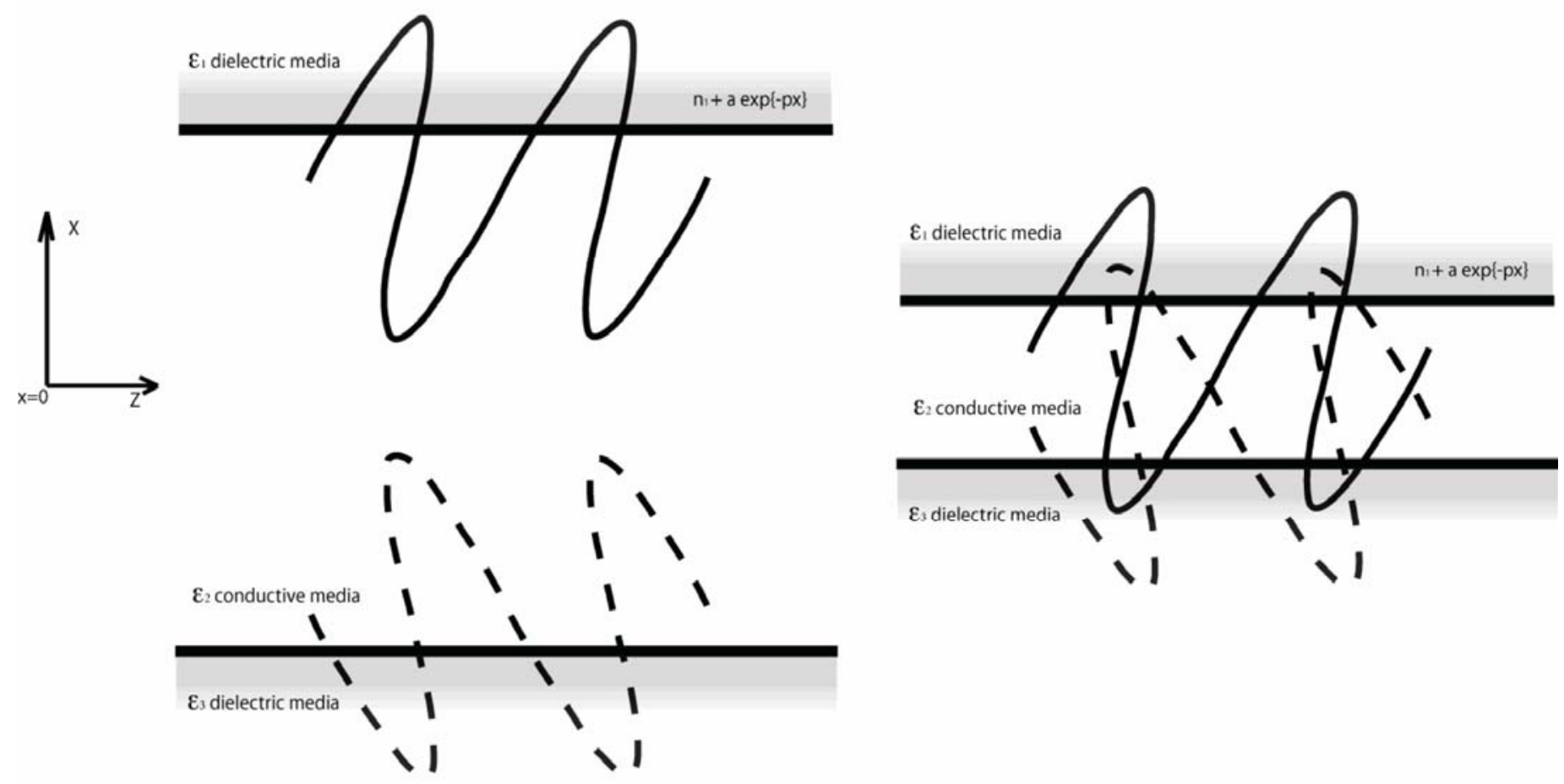

FIG. 1 Scheme to describe the interaction between two surface plasmon modes. In the left side the thickness of the metal film is large enough that no plasmon interaction is generated. In the right side the interaction between two plasmon propagating on each side of a thin film modifies the dispersion relation function. In both cases, the continue line represents an elementary surface plasmon generate on $\varepsilon_{1}-\varepsilon_{2}$ interface, the dashed line represents an elementary surface plasmon generated on $\varepsilon_{2}-\varepsilon_{3}$ interface.

surface plasmon mode propagates short distances, vanishing completely in about $100 \mu \mathrm{m}$, this represent a serious limitation in the application of the elementary plasmon modes [5]-[7].

Related to this electromagnetic mode is the charge motion of the free electrons that oscillates longitudinally and transversally to the surface. A geometrical interpretation of charge oscillations in the plasmon phenomena is that these oscillations are determined by the trajectory of the electric field, whose geometry can be obtained establishing an analogy with the classical polarization model. It is easy to show that the trajectory of the electric field on the $x-z$ plane is given by

$\frac{E_{x}^{2}}{a_{1}^{2}}+\frac{E_{z}^{2}}{\left(\alpha a_{1} /|\beta|\right)^{2}}-\frac{2 E_{x} E_{z}}{a_{1}^{2} \alpha /|\beta|} \cos \delta_{0}=\exp \{-2 \alpha x\} \sin ^{2} \delta_{0}$.

Where $\beta=|\beta| \exp \{i \delta\}$ and $\delta_{0}=\delta-\pi / 2$. It must be noted that $\delta$ depends of the permittivity associated with the metal media. From Eq. 3 we deduce that a large $\beta$ is desirable in order to decrease the interaction of the $k$-component, of the electric field with the surface, obtaining electromagnetic waves capable of propagating long distances. The amplitude of $k$-component depends inversely on the dispersion relation function $\beta$ and we consider a "more homogeneous" surface plasmon mode. In general Eq. 4 represents an ellipse, and large $\beta$ means that the mayor axis becomes closer to the $x$-axis. Then we have geometrically that the eccentricity of the ellipse is a good measure of the homogeneity of the surface plasmon mode.

\section{LONG-RANGE PLASMON MODES}

Here we describe the generation of long-range surface plasmon modes propagating on a interface dielectric-metal thin film considering the refractive index as a decreasing exponential function which allows us the study of resonance effects, the analysis is performed using the coupling mode theory. In order to obtain a long-range plasmon mode we need to adapt the dispersion relation function $\beta$, this analysis has been reported by other authors in several configurations [11]-[13]. Resonance effects emerge when the interaction with other surface plasmon is allowed, modifying the trajectory of charge oscillations. The new surface plasmon modes has an amplitude depending of $z$-coordinate. The system under analysis is sketched (see Figure 1).

The refractive index for the interface is approximated by

$$
n=n_{1}+\alpha \exp \{-p x\} \quad x \geq d
$$

in last equation $a$ is a small constant that is determined by the combination of the materials involved and $2 d$ is the thickness of the film. For the region $x \geq d$, we assume a similar expression for the refractive index. It must be noted when $x \rightarrow \infty$ the refractive index so expressed recovers the classic expression for a dielectric media. The reason to pro- 
pose the expression for the refractive index comes from the fact that a beam propagating parallel and sufficient closed to a flat surface is bended this means that the refractive index in the surface neighbourhood looks like no more constant, then we have that the exponential term allows us to model the surface effects.

The plasmon field has a vector representation, where each scalar term must satisfy a Helmholtz equation of the form

$$
\nabla^{2} \varphi+K_{0}^{2}\left(n_{1}^{2}+2 n_{1} a \exp \{-p x\}\right) \varphi=0,
$$

in Eq. 6, a term in the form $a^{2} \exp \{-2 p x\}$ has been disregarded by smallness. The coupling mode theory is used to propose a solution in the dielectric-metal interface of the form

$$
\begin{array}{r}
\varphi(x, y, z)=A_{1}(z) \exp \left\{-\alpha_{1}|x-d|\right\} \exp \left\{i \beta_{1} z\right\}+ \\
A_{2}(z) \exp \left\{-\alpha_{2}|x-d|\right\} \exp \left\{i \beta_{2} z\right\} .
\end{array}
$$

This solution is valid in the region near to the interface sketched as shadow region in Figure 1. Selecting the appropriate sign in the phase terms and by direct substitution, we find that the differential equations for the amplitude functions $A_{l, 2}(z)$ satisfies

$$
\begin{aligned}
& i \beta_{1} \frac{\partial A_{1}}{\partial z}+2 a n_{1} A_{2} \exp \left\{-\left(\alpha_{1}+\alpha_{2}\right) d\right\} \exp \left\{i\left(\beta_{2}-\beta_{1}\right) z\right\}=0, \\
& i \beta_{2} \frac{\partial A_{2}}{\partial z}+2 a n_{1} A_{1} \exp \left\{-\left(\alpha_{1}+\alpha_{2}\right) d\right\} \exp \left\{-i\left(\beta_{2}-\beta_{1}\right) z\right\}=0 .
\end{aligned}
$$

Where the second order derivatives have been omitted, this is the slow envelope condition [14],

$$
\frac{\partial^{2} A_{1}}{\partial z^{2}} \approx \frac{\partial^{2} A_{2}}{\partial z^{2}} \approx 0
$$

This condition is close related with the curvature of the profile solution, decreasing the interaction of the electromagnetic field with the surface, which justifies on a physical basis the possibility to generate long range plasmon modes.

The parameter $p$ in the expression for the refractive index Eq. 5 is matched with the $\alpha_{i}$-parameter by means of the relation $p$ $=\alpha_{1}+\alpha_{2}$, which is the required condition for the resonance between the two surface plasmon modes, this condition is analogous to the Bragg condition [14]. The resonance effect modifies the trajectory of the oscillation of the charges changing in consequence the dispersion relation function $\beta$.

A symmetrical configuration is obtained when the permittivity in both sides of the metal thin film has the same value, we have that $\beta_{1}=\beta_{2}=\beta ; \alpha_{1}=\alpha_{2}=\alpha$ and the system of differential equations takes the simplest form

$$
\begin{aligned}
& i \beta \frac{\partial A_{1}}{\partial z}+2 a n_{1} A_{2} \exp \{-2 \alpha d\}=0, \\
& i \beta \frac{\partial A_{2}}{\partial z}+2 a n_{1} A_{1} \exp \{-2 \alpha d\}=0
\end{aligned}
$$

whose solution is

$$
\begin{array}{r}
A_{1,2}(z)=c_{1,2} \exp \left\{i\left(\frac{2 a n_{1}}{\beta} \exp \{-2 \alpha d\}\right) z\right\}+ \\
c_{2,2} \exp \left\{-i\left(\frac{2 a n_{1}}{\beta} \exp \{-2 \alpha d\}\right) z\right\},
\end{array}
$$

where $c_{1,2}, c_{2,2}$ are arbitrary constants. Then we have that the expression for long-range plasmon modes is of the form

$$
\begin{aligned}
& \varphi(x, z)=c \exp \{-\alpha x\} \exp \\
& \left\{i z \frac{\omega}{c}\left(\left(\frac{\varepsilon_{1} \varepsilon_{2}}{\varepsilon_{1}+\varepsilon_{2}}\right)^{1 / 2}+2 a n_{1}\left(\frac{\varepsilon_{1} \varepsilon_{2}}{\varepsilon_{1}+\varepsilon_{2}}\right)^{-1 / 2} \exp \{-2 \alpha d\}\right)\right\} .
\end{aligned}
$$

Where the modified dispersion relation function is given by

$$
\beta=\frac{\omega}{c}\left\{\left(\frac{\varepsilon_{1} \varepsilon_{2}}{\varepsilon_{1}+\varepsilon_{2}}\right)^{1 / 2}+2 a n_{1}\left(\frac{\varepsilon_{1} \varepsilon_{2}}{\varepsilon_{1}+\varepsilon_{2}}\right)^{-1 / 2} \exp \{-2 \alpha d\},\right.
$$

Is easy to show that in this representation the sum of the imaginary parts is lower than the imaginary part of the relation dispersion function for semi-infinite media, given us the ability to increase the length propagation of the surface plasmon mode. This relation has a view similar to the one obtained in references [11, 15]. However, our expression allows us to associate a geometric interpretation for the charge oscillations [16, 17] sketched (see Figure 1). In addition, the coefficient for the decreasing exponential term depends on the refractive index parameters which are necessary to describe the surface effects. These effects are relevant when two surfaces are sufficiently near each other or a particle is placed in the surface neighborhood. It must be noted that $a \neq 0$, however, when $d \rightarrow \infty$ the obtained dispersion relation function recovers the form for a semi-infinite media.

\section{INTERACTION BETWEEN TWO LONG-RANGE PLASMON MODES: COHERENT CASE}

Eq. 12 is the expression for a component of the electric field propagating along the $z$-coordinate. In order to generate the interference between two long-range plasmon modes, it is necessary to represent the long-range plasmon mode propagating on the $y-z$ plane. The corresponding expression can be obtained by means of a rotation respect the $x$-axis, having the transformation of coordinates $x \rightarrow x ; z \rightarrow z \cos \theta \pm y \sin \theta$. The physical configuration to generate the interference between two long-range plasmon modes is similar to Young's experiment that consists in two sub-wavelength apertures on a conductive thin film. From each aperture emerges a longrange plasmon mode, the expression for the coherent interference is

$$
\begin{aligned}
& E(x, y, z)= \\
& (\hat{i} a+\hat{k} b \cos \theta+\hat{j} b \sin \theta) \exp \left\{-\alpha_{1} x\right\} \exp \{i \beta(z \cos \theta+y \sin \theta)\}+ \\
& (\hat{i} a+\hat{k} b \cos \theta-\hat{j} b \sin \theta) \exp \left\{-\alpha_{1} x\right\} \exp \{i \beta(z \cos \theta-y \sin \theta)\} \\
& =(2(\hat{i} a+\hat{k} b \cos \theta) \cos (\beta y \sin \theta)+\hat{j} 2 i b \sin \theta \sin (\beta y \sin \theta)) \times \\
& \exp \left\{-\alpha_{1} x\right\} \exp \{i \beta z \cos \theta\} .
\end{aligned}
$$


With $\beta$ given in Eq. 13 Eq. 14 can be considered as a plasmon diffraction free mode because the field intensity for long distances of propagation remains unchanged, this same approximation was used by Durnin [9], then we have that the field intensity is

$I(x, y, z)=$

$4\left(a^{2}+b^{2} \cos ^{2} \theta\right) \cos ^{2}(\beta y \sin \theta)+4 b^{2} \sin ^{2} \theta \sin ^{2}(\beta y \sin \theta) \times$

$\exp \left\{-2 \alpha_{1} x\right\} \exp \{-2 \operatorname{Im}(\beta) z\}$.

For small angle $\theta$, the expression for generalized plasmon modes can be approximated as

$$
\begin{aligned}
& E(x, z)= \\
& (\hat{i} a+\hat{k} b) \cos (\beta y \sin \theta) \exp \left\{-\alpha_{1} x\right\} \exp \{i \beta \cos \theta z\},
\end{aligned}
$$

and it is considered as the expression for a generalized plasmon mode completely coherent in the paraxial approximation. Using the fact that $a$ and $b$ may be complex numbers, the field intensity distribution can be rewritten as

$$
\begin{aligned}
& I(x, y, z)= \\
& c^{2} \cos ^{2}(\beta y \sin \theta+\eta) \exp \left\{-2 \alpha_{1} x\right\} \exp \{-2 \operatorname{Im}(\beta) z\},
\end{aligned}
$$

where $\eta$ is a phase term. Up to now, we have that sinusoidal surface plasmon modes represent an expression for a completely coherent generalized surface mode. It must be noted that Eq. 17 is a parametric representation for surface plasmon modes. The parameters $\theta$ and $\eta$ allows to incorporate partially coherent features as it is described in the following section.

In Figure 2 we show the field intensity distribution for a sinusoidal plasmon mode, the parameters employed in the numerical simulation are a gold film with thickness of $20 \mathrm{~nm}$, the wavelength is $500 \mathrm{~nm}$, the substrate has a refractive index of 2 , the range of values for $p$ is $0.01 \mathrm{~nm}^{-1}$ to $0.023 \mathrm{~nm}^{-1}$, These values were obtained using the fact that a surface plasmon mode can propagate $100 \mathrm{~nm}$. into the dielectric media, according to Raether [1]. With these parameters we expect that the plasmon mode propagates between $500 \mu \mathrm{m}$ to $1000 \mu \mathrm{m}$.

\section{GENERALIZED SURFACE PLASMON MODES: PARTIALLY COHERENT CASE}

It is a well known fact that the spatial frequency representation for diffracting free beams propagating in free space must be on a circle. The corresponding frequency representation for a generalized surface plasmon mode consists of two points as may be deduced from Eq. 14. These two points are obtained by intersecting the frequency circle with the interface plane. These are the reasons why sinusoidal modes represent generalized completely coherent surface plasmon modes. Additional details concerning the frequency representation for diffracting free beams can be found in references [7]-[10].

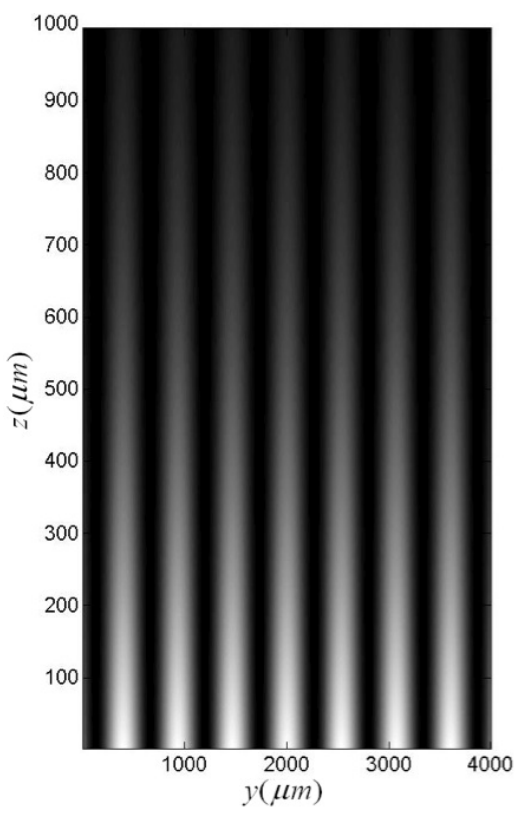

FIG. 2 Sinusoidal profile of the field intensity distribution generated by interfering two long range plasmon modes propagating on $x=0$ plane.

The partially coherent surface plasmon mode can be obtained by means of an ergodic superposition of sinusoidal plasmon modes, the parameter $\theta$ in Eq. 17 is a random variable with probability density function $\rho(\theta)$. The mean field intensity distribution function is

$$
\begin{aligned}
& \langle I(x, y, z)\rangle= \\
& \exp \left\{-\alpha_{1} x\right\} \exp \{-2 \operatorname{Im}(\beta) z\} \int C(\theta) \cos ^{2}(\beta y \sin \theta+\eta) \rho(\theta) d \theta .
\end{aligned}
$$

In Figure 3 we show the computational simulations for the incoherent superposition of sinusoidal plasmon modes for different $\rho(\theta)$, using the same parameters as in Figure 2. In Figure $3 a$, the probability density function is uniform with $\eta=0$ and a $J_{0}$ Bessel surface plasmon mode is obtained, this mode propagates about $500 \mu \mathrm{m}$. In Figure 3b, the probability density function is Gaussian, the profile along the $y$ coordinate is Gaussian, and its length of propagation is approximately $700 \mu \mathrm{m}$ exceeding the Bessel case. Finally in Figure $3 c$ the probability density function is uniform and by setting the value $\eta=\Pi / 2$, the incoherent convergence tends to a Dark Hollow surface plasmon mode, in this case the length propagation is about $50 \mu \mathrm{m}$. This is easily understood because the energy is distributed on a bigger area. In all cases the dispersion relation function is given by Eq. 13. In spite of all the modes have the same dispersion relation function, the length of propagation can be controlled with the superposition of modes. The explicit calculations for the incoherent convergence are shown in appendix A.

Controlling the phase parameter $\eta$ also as the probability density function for the relative separation between the apertures we are able to control the intensity distribution as it is shown in Figures 3. This is an implication of the partially coherent features. Physically implies to control the length of propagation of the surface plasmon synthesized. 


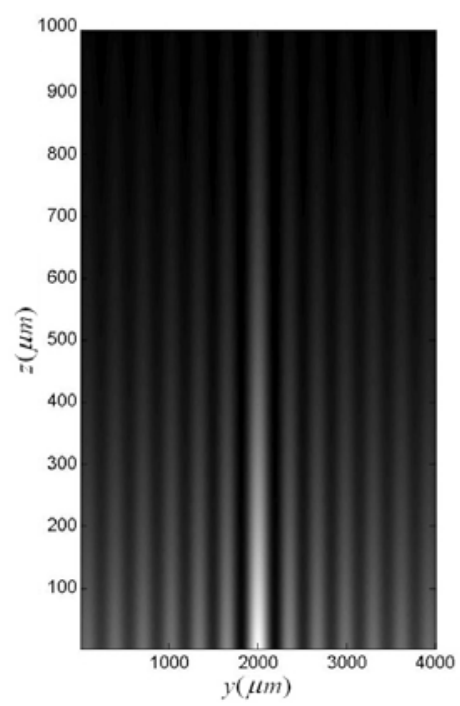

a)

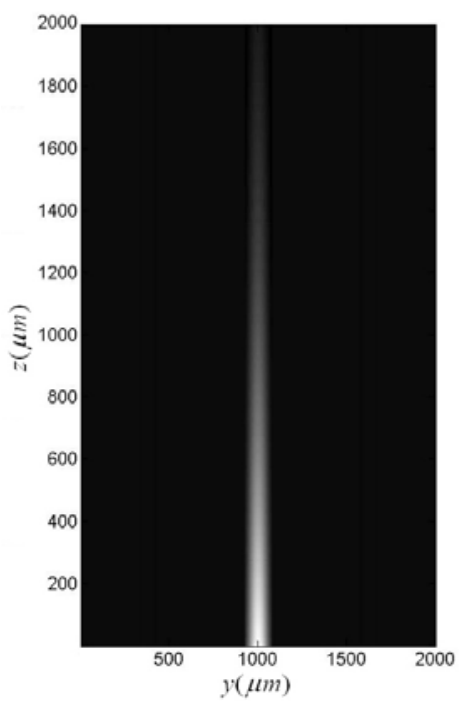

b)

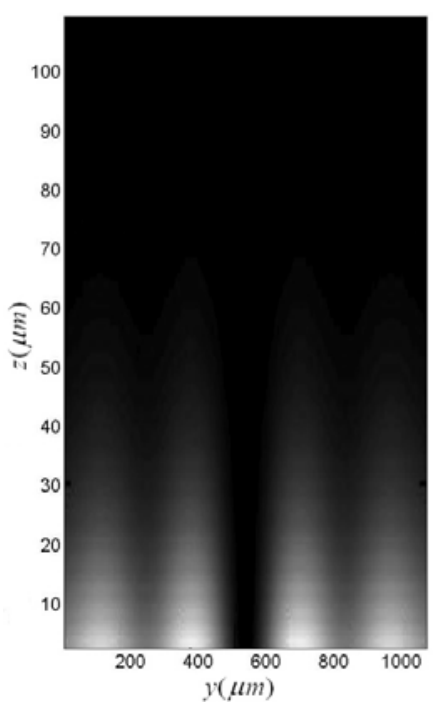

c)

FIG. 3 Mean field intensity distributions on $y$-z plane for: a) $J_{0}$ Bessel surface plasmon mode, b) Gaussian surface plasmon mode and c) Dark Hollow surface plasmon mode.

For the experimental implementation [16, 17], a possible set up consists in a metallic thin film with two sub-wavelength apertures deposited on an elastic polymer substrate. Compressing mechanically the polymer along the $y$-axis that connects the apertures we are able to control the relative separation between the apertures. This configuration allows us to implement a generalized surface plasmon as optical tweezers, in order to avoid the drag forces, the apertures are illuminated from the bottom of the substrate and the nanoparticles are deposited over the metallic film, in this way we have only a surface plasmon-nanoparticle interaction. The experimental details will be presented in a forthcoming paper.

\section{CONCLUSIONS}

By proposing a decreasing exponential representation for the refractive index in the neighbourhood of a dielectricconducting media, and using coupling mode theory we found a new expression for the dispersion relation function that allows the synthesis of long-range plasmon modes. The interference between these modes generates a completely coherent generalized plasmon mode whose profile is cosine like. The sinusoidal plasmon modes were implemented to generate a new variety of modes, by considering the spatial period as a random process. The energy convergence of the sinusoidal modes is controlled by means of the probability density function, this convergence offers a more versatile way to control the propagation length. As an example of this convergence, we synthesized $J_{0}$ Bessel, Gaussian and Dark Hollow surface plasmon modes, which present partially coherent features, until our knowledge this is the first time that this kind of surface plasmon modes have been reported. These modes offer applications for the synthesis of plasmonic tweezers, trapping and confinement of particles, tuneable resonance effects, etc. An important topic that remains to be studied is the analysis of arbitrary partially coherent plas- mon fields which can be represented as a superposition of generalized plasmon modes, this representation allows the study of the partially coherent features establishing the VanCittert-Zernike theorem for plasmonic fields. This analysis will be presented in a forthcoming paper. One of the authors (GMN) is very grateful to the "Consejo Nacional de Ciencia y Tecnologia, CONACyT" by the support through project research No. 47325.

\section{APPENDIX}

The expression for the mean field intensity associated with a sinusoidal surface plasmon mode rewritten Eq. 18 is

$$
\begin{aligned}
& \langle I(x, y, z)\rangle=\exp \left\{-\alpha_{1} x\right\} \exp \{-2 \operatorname{Im}(\beta) z\} \\
& \int C(1+\cos 2 \beta y \sin \theta+\eta) \rho(\theta) d \theta,
\end{aligned}
$$

where $C$ is a constant, If the probability density function $\rho(\theta)$ is uniform in the interval $-\pi / 2 \leq \theta \leq \pi / 2$, and considering $\eta=0$, we have that the mean field intensity is given by a Jo Bessel function

$$
\begin{aligned}
& \langle I(x, y, z)\rangle= \\
& \exp \left\{-\alpha_{1} x\right\} \exp \{-2 \operatorname{Im}(\beta) z\}\left(1+J_{0}(2 \beta y)\right),
\end{aligned}
$$

where no relevant terms has been omitted. We used the result

$$
J_{0}(x)=\frac{2}{\pi} \int_{0}^{\pi / 2} \cos (x \sin \theta) d \theta
$$

To justify the mean field intensity for the dark hollow surface plasmon mode we consider $\eta=\pi / 2$ and the Eq. A.1 takes the form 


$$
\begin{aligned}
& \langle I(x, y, z)\rangle \\
& =\exp \left\{-\alpha_{1} x\right\} \exp \{-2 \operatorname{Im}(\beta) z\} \int(1-\cos 2 \beta y \sin \theta) \rho(\theta) d \theta \\
& =\exp \left\{-\alpha_{1} x\right\} \exp \{-2 \operatorname{Im}(\beta) z\}\left(1-J_{0}(2 \beta y)\right) .
\end{aligned}
$$

This result presents a reverse contrast in the mean field intensity distribution respect to the $J_{0}$ Bessel surface plasmon mode.

To explain the field intensity distribution for a Gaussian surface plasmon mode we consider $\eta=0$ and use the probability density function $\rho(\theta)=a \exp -\theta^{2} / 2$ in the interval $-\pi / 2 \leq \theta \leq \pi / 2$. Considering the approximation, we have that Eq. A.1 takes the form

$$
\begin{aligned}
& \langle I(x, y, z)\rangle \\
& =\exp \left\{-\alpha_{1} x\right\} \exp \{-2 \operatorname{Im}(\beta) z\} \times \\
& a\left(\begin{array}{l}
\left.1+\frac{1}{2} \int \exp \{i 2 \beta y \theta\} \exp \left\{-\frac{\theta^{2}}{2}\right\} d \theta\right), \\
+\int \exp \{-i 2 \beta y \theta\} \exp \left\{-\frac{\theta^{2}}{2}\right\} d \theta
\end{array}\right),
\end{aligned}
$$

this can be approximated as the Fourier transform of a Gaussian function, justifying in this way the mean field intensity described in Figure 3b.

\section{References}

[1] H. Raether, "Surface plasmons on smooth and rough surfaces and on gratings" Springer Tr. Mod. Phys. 111, 1-7 (1988).

[2] M. Osawa, "Near-field optics and surface plasmon polaritons" Top. Appl. Phys. 81, 163-187 (2001).

[3] M. Osawa, M. Kuramitsu, A. Hatta, W. Suetaka, "Electromagnetic effect in enhanced infrared absorption of absorbed molecules on thin metal films" Surf. Sci. Lett. 175, L787-L793 (1986).

[4] S. Sato, K. Kamada, M. Osawa, "Surface-enhanced IR absorption (SEIRA) on small Pt particles deposited on an island Au films" Chem. Lett., 15-16 (1999).

[5] I.I. Smolyaninov, D.L. Mazzoni, C.C. Davis, "Imaging of surface plasmon scattering by lithographically created individual surface defects" Phys. Rev. Lett. 77, 3877-3880 (1996).

[6] I.I. Smolyaninov, J. Elliot, A. Sayats, C.C. Davis, "Quantum fluctuations of the refractive index near the interface between a metal and a nonlinear dielectric" Phys. Rev. Lett. 94, 57401-57403 (2005).

[7] G. Martinez Niconoff, J.A. Sanchez-Gil, H.H. Sanchez-Hernandez and A. Perez-Leija, "Self-imaging and caustics in twodimensional surface plasmon optics" Opt. Comm. 281, 2316-2320 (2008).

[8] L. Mandel and E. Wolf, Optical Coherence and Quantum Optics (Cambridge University Press, Cambridge, 1995).

[9] J. Durnin, "Exact solutions for nondiffracting beams I. The scalar theory" J. Opt. Soc. Am A. 4, 651-654 (1987).

[10] G. Martínez Niconoff, J.C. Ramírez San Juan. J. Munoz-Lopez and P. Martínez Vara, "Incoherent convergence of diffraction free fields" Opt. Comm. 275, 10-13 (2007)

[11] J.J. Burke and C.I. Stegeman and T. Tamir, "Surface-polariton-like waves guided by thin, lossy metal films" Phys Rev. B 33, 51865201 (1986).

[12] A.R. Davoyan, I.V. Shadridov and Y.S. Kivshar, "Quadratic phase matching in nonlinear plasmonic nanoscale waveguides" 0 pt. Exp. 17, 20063-20068 (2009).

[13] F. Yang, J.R. Sambles, G.W. Bradberry, "Prism coupling to longrange coupled-surface modes" J. Mod. Optic 38, 707-717 (1991).

[14] P. Yeh, Introduction to Photo-refractive Nonlinear Optics (Wiley, New York, 1993).

[15] I.I. Smolyaninov, J. Elliot, A. Sayats and C.C. Davis, "Far-field optical microscopy with a nanometer-scale resolution based on in the in-plane image magnification by surface plasmon polaritons", Phys. Rev. Lett. 94, 57401 (2005).

[16] C.H. Gan, G. Gbur and T.D. Visser, "Surface plasmons modulate the spatial coherence of light in Young's interference experiment" Phys. Rev. Lett. 98, 043908 (2007).

[17] E. Altewischer, M.P. van Exter and J.P. Woerdman "Plasmonassisted transmission of entangled photons" Nature 418, 304306 (2002). 